\title{
Dynamic Resource Sharing in 5G with LSA: Criteria-Based Management Framework
}

\author{
Zhaleh Sadreddini (D), ${ }^{1}$ Pavel Masek $\left(D,{ }^{2,3}\right.$ Tugrul Cavdar, ${ }^{1}$ \\ Aleksandr Ometov $\odot{ }^{1}{ }^{4}$ Jiri Hosek $(1)^{2,3}$ Irina Gudkova, ${ }^{3,5}$ and Sergey Andreev ${ }^{4}$ \\ ${ }^{1}$ Department of Computer Engineering, Karadeniz Technical University, Trabzon, Turkey \\ ${ }^{2}$ Department of Telecommunications, Brno University of Technology, Brno, Czech Republic \\ ${ }^{3}$ Applied Probability and Informatics Department, Peoples' Friendship University of Russia (RUDN University), Moscow, Russia \\ ${ }^{4}$ Laboratory of Electronics and Communications Engineering, Tampere University of Technology, Tampere, Finland \\ ${ }^{5}$ Federal Research Center "Computer Science and Control" of the Russian Academy of Sciences, Moscow, Russia
}

Correspondence should be addressed to Pavel Masek; masekpavel@feec.vutbr.cz

Received 27 July 2017; Revised 18 January 2018; Accepted 5 March 2018; Published 24 May 2018

Academic Editor: Oscar Esparza

Copyright (c) 2018 Zhaleh Sadreddini et al. This is an open access article distributed under the Creative Commons Attribution License, which permits unrestricted use, distribution, and reproduction in any medium, provided the original work is properly cited.

\begin{abstract}
Owing to a steadily increasing demand for efficient spectrum utilization as part of the fifth-generation (5G) cellular concept, it becomes crucial to revise the existing radio spectrum management techniques and provide more flexible solutions for the corresponding challenges. A new wave of spectrum policy reforms can thus be envisaged by producing a paradigm shift from static to dynamic orchestration of shared resources. The emerging Licensed Shared Access (LSA) regulatory framework enables flexible spectrum sharing between a limited number of users that access the same frequency bands, while guaranteeing better interference mitigation. In this work, an advanced user satisfaction-aware spectrum management strategy for dynamic LSA management in 5G networks is proposed to balance both the connected user satisfaction and the Mobile Network Operator (MNO) resource utilization. The approach is based on the MNO decision policy that combines both pricing and rejection rules in the implemented processes. Our study offers a classification built over several types of users, different corresponding attributes, and a number of MNO's decision scenarios. Our investigations are built on Criteria-Based Resource Management (CBRM) framework, which has been specifically designed to facilitate dynamic LSA management in $5 \mathrm{G}$ mobile networks. To verify the proposed model, the results (spectrum utilization, estimated Secondary User price for the future connection, and user selection methodology in case of user rejection process) are validated numerically as we yield important conclusions on the applicability of our approach, which may offer valuable guidelines for efficient radio spectrum management in highly dynamic and heterogeneous $5 \mathrm{G}$ environments.
\end{abstract}

\section{Introduction}

1.1. Background on Spectrum Scarcity and Sharing. The tremendous growth in mobile data volume has become a key driver for the development of emerging fifth-generation $(5 \mathrm{G})$ cellular systems. As it is forecasted by Cisco [1], the required capacity will be challenged by the growing utilization of mobile devices (e.g., smartphones) that access a diverse set of services and applications in order to manage increasing amounts of data traffic. Following this trend, certain research and development considerations on the next-generation wireless systems might be regarded in the light of steady increase of the overall demand for radio spectrum as well as the deployment of a variety of technologies that compete to utilize the shared frequency bands [2]. The traditional distinction between different categories of applications, such as data services, voice communications, and broadcasting, becomes blurred due to convergence in functionality of these services delivered by new technologies $[3,4]$.

At the same time, radio spectrum has transformed into a critical resource from economic, cultural, and societal perspectives $[5,6]$. Its scarcity has become a major limiting factor in particular frequency ranges, spanning $100 \mathrm{MHz}$ to $6 \mathrm{GHz}$, with desired propagation characteristics for a 
wide range of static spectrum users (e.g., military, radar, TV broadcasting, and medical and event production) [7, 8]. Up to now, mobile operators preferred to follow the traditional exclusive spectrum access regime when offering their services. However, this led to utilizing large portions of radio spectrum only sporadically, with high space, time, and frequency variations in usage that ranged from $15 \%$ to $85 \%$ [9]. As the needs for extra spectrum have been growing over the past decades, alternative solutions were proposed, especially for exploiting small fragments of spectrum that are seldom used by their license holders.

With respect to spectrum management, several concepts have been developed and deployed over the years [10, 11]. Accordingly, the benefits of spectrum sharing are twofold. First, it allows improvements in spectrum utilization and second it can provide additional capacity for the users who require more spectrum for their services. A wide range of spectrum sharing schemes can facilitate the utilization of different frequency bands (both licensed and unlicensed), as adopted by various carrier-grade service providers. The deployment of spectrum sharing schemes is subject to meeting a set of predefined regulations and requirements. It can also involve dissimilar coordination techniques. Actively considered by telecom operators, dynamic spectrum sharing can enable intertechnology coexistence in licensed bands [1214]. Currently, the efficiency of spectrum sharing mechanisms remains limited by preset priority constraints of the primary license holder. Here, the broader concept of spectrum sharing refers to equal spectrum access rights but may overlook economical aspects and user satisfaction considerations [15].

1.2. Spectrum Sharing with Licensed Shared Access. To overcome the pressing effects of spectrum fragmentation, a demand for novel frameworks allowing for efficient sharing of available but underutilized spectrum emerges. A novel Licensed Shared Access (LSA) [16, 17] concept enables advanced spectrum sharing between a limited number of end-users with at least two entities: the incumbent (i.e., the current holder of the spectrum usage rights) and the LSA licensee (i.e., the temporary user of spectrum, which could be a Mobile Network Operator, MNO) [18]. More specifically, the LSA framework [19] permits for controlled spectrum sharing between a Primary User (PU) and a Secondary User (SU) both having access to a portion of spectrum at a given location and time [20, 21], as it is demonstrated in Figure 1.

The corresponding LSA sharing agreements need to guarantee high uniformity in terms of spectrum access for all involved participants. To this end, satisfying end-user service requirements is a major goal in the mobile operator's system design. The MNOs should maximize the active SUs (ASUs) satisfaction by shifting the emphasis from Quality of Service (QoS) to Quality of Experience (QoE). Therefore, decisions made by the MNOs play an important role in the discussed ecosystem and consideration of appropriate parameters affects the overall network performance. To this end, the network parameters and user attributes should be evaluated jointly for the optimized MNO decision-making $[22,23]$, which becomes the focus of this work.

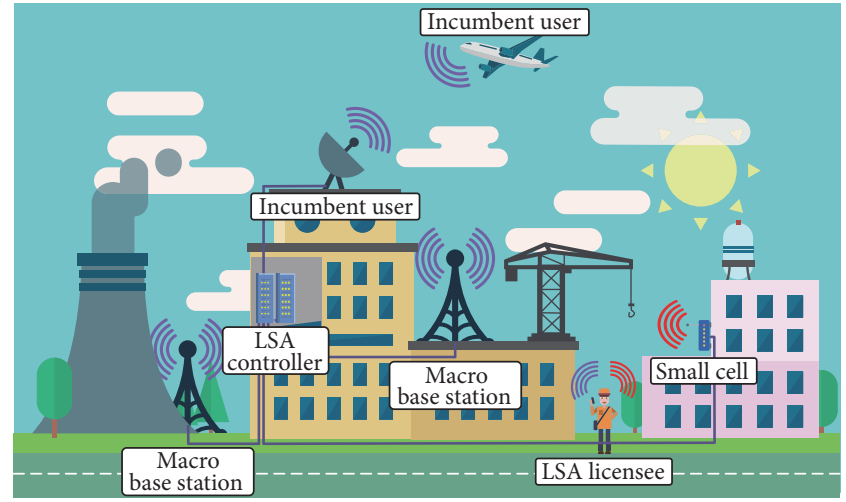

FIGURE 1: Scenario of LSA framework operation.

The rest of the paper is organized as follows. Section 2 provides an overview of existing solutions. Next, Section 3 describes the proposed system model for the user experienceaware spectrum management together with developed DecisionBasedRejectionPolicy algorithm. Further, in Section 4, the selected use-cases are discussed. In Section 5, the obtained simulation results are described. Finally, the lessons learned and conclusions are summarized in Section 6. See "List of Frequently Used Acronyms" in Nomenclature.

\section{Related Work and Our Focus}

The emerging LSA framework requires MNOs to develop efficient spectrum management strategies in order to increase the network capacity and provide high user experience levels to the PUs and SUs. As a result, higher MNO revenues are expected to be reached. As it has been already demonstrated in [24], decision-making is one of the main functions of the radio spectrum management process allowing the MNOs to determine the radio parameters that control the spectrum utilization.

In [25], dynamic billing and pricing strategy is introduced for the LTE networks. The calculated resource cost varies according to the user category and congestion threshold specified by the MNO. The considered parameter of the end-users is priority class (e.g., gold, silver, or bronze). In [24], the authors proposed an Adaptive Decision-making Optimization Scheme (ADMS) for Cognitive Radio Network (CRN) with multiple subcarriers. All of the variables in the fitness function are related to the network parameters. Besides that, the authors considered the weight of parameters in the fitness function being adaptive. However, the weight adaptive function is not defined in addition to the use of fixed weights in the simulation results.

The work in [26] provides a connectivity management platform for efficient Wi-Fi offloading in heterogeneous networks. Technique for Order of Preference by Similarity to Ideal Solution (TOPSIS) with the consideration of two attributes, Received Signal Strength Indicator (RSSI) and number of connected users, was applied. The spectrum utility model was developed in [27] utilizing the multiattribute utility theory to form the user's decision model. The corresponding model allows a user to balance the attributes, such 
as channel capacity, monetary cost, and interference potential. Further, in [28], the impact of end-user decision-making on pricing and management of radio resources in CRN was studied.

In this work, Criteria-Based Resource Management (CBRM) framework for LSA is presented as a trade-off between the MNO's decision on network management and the QoE of users. In our previous research [29], Instant Overbooking Framework for Cognitive Radio (IOFCR) was formulated to enable efficient management of spectrum in CRN. Three different pricing policies for the booking period have been considered. In this study, we implement CBRM with respect to the crucial user and network attributes in LSA. We utilize criteria-based pricing and rejection policies, where the weights of all the parameters are updated periodically and automatically based on the MNO's decision and following the given QoS requirements as well as the spectrum usage ratio. The user satisfaction can be maximized by manipulating resource allocation via adaptive pricing and rejection policies based on the QoE.

In more detail, if the $\mathrm{MNO}$ is not able to provide ASUs with adequate QoE, the rejected history ratio for the rejected ASUs (RASUs) will be high. The rejected history ratio as a Compensation Cost (CC) has strong impact on the pricing policy and it could convince RASUs to request spectrum resources from the network as RSUs in the nearest future. Following our previous work [30], we utilize two server pool systems: they categorize RSUs based on the QoE via the rejected history parameters. Hence, we study usercentric QoE-based resource allocation problem with several MNO's decisions, several user criteria, and different classes of services. Our simulation results indicate the spectrum situation in off-peak and peak hours.

\section{System Model}

In this section, the Total Network Revenue aspect is detailed through (i) resource block leasing, (ii) compensation paid to the rejected ASUs, (iii) spectrum utilization, (iv) number of accepted and denied RSUs, (v) number of rejected and finished ASUs (RASUs/FASUs), and (vi) average service time for both RASUs and FASUs.

To provide a comprehensive understanding of the proposed CBRM framework for LSA, we continue this section by detailing the developed model. Attention is specifically paid to several types of customers, different user attributes, and several MNO decision use-cases, while highlighting the important simulation results and discussing the strategies to reach higher QoE levels for ASUs.

A detailed description of the proposed system model in this section is divided into 3 subsections. (i) Network Architecture and Scenario: in this subsection, the entities of the proposed LSA system and the interactions between them are introduced. (ii) Utilized Attributes: in this part, with respect to the impact of decision-making on pricing and management of URB in the CBRM framework for LSA, important used attributes for the proposed system model are defined. After utilizing the attributes for SUs, the total number of RSUs, ASUs, FASUs, and RASUs can be shown

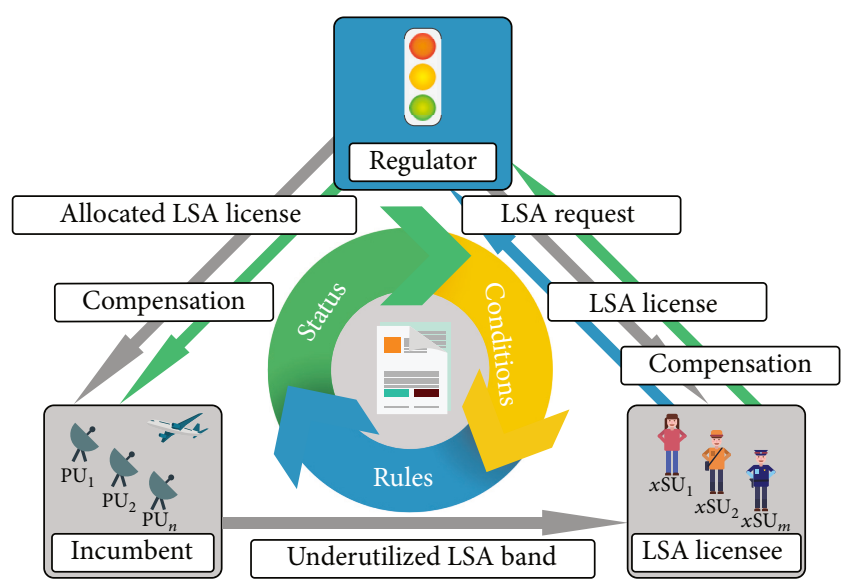

Figure 2: Proposed system model for LSA operation.

in one list as $L_{x \mathrm{SU}}\left(t_{i}\right)$. At time instant $t_{i}$, depending on the URB number, MNO is faced with the four main use-cases, which are described in this subsection. (iii) Criteria-Based Rejection Model: in case of $0 \leq \mathrm{URB}(t i)<|\mathrm{LPU}(t i)|$, MNO should reject the appropriate ASUs. In this subsection, DecisionBasedRejectionPolicy is implemented by AHP at the side of the LSA licensee to reject the appropriate ASUs. Finally, the CC is calculated for the rejected ASUs. In order to obtain higher network revenue, while motivating users to request the spectrum resources more frequently, the expected price for the rejected ASUs is calculated based on the defined RR.

3.1. Network Architecture and Scenario. In order to represent the real-world use-cases, we utilize LTE-Advanced (Rel. 10) network infrastructure with the implemented LSA framework, as detailed in our previous work [30]. The interaction between the corresponding entities of the LSA system is depicted in Figure 2. The considered network architecture consists of the following building blocks:

(i) Incumbent: LSA is the controlled shared access framework based on an exclusive regime of radio spectrum sharing among the incumbents, that is, PUs, that have the right to commercially exploit a given portion of wireless spectrum (cellular band).

(ii) LSA licensee: licensed users that lease the incumbent's spectrum band, which can potentially be used when a permission is granted. The leased resources (i.e., resource blocks) are further offered to the LSA Licensee's SUs under the control of the entity responsible for granting permissions over the cellular band (i.e., LSA controller). In this work, we assume four different states of SUs (i.e., $x \mathrm{SU}_{1, m}$ ):

(1) Requesting Secondary Users (RSUs): at time instant $t_{i}$, there are RSUs that send spectrum availability requests to the LSA licensees (modeling is detailed in our previous paper [29]). The LSA controller monitors the sharing activities in the cellular network and the corresponding 
decisions (accept or reject the RSUs) are made based on spectrum availability provided by incumbents to the LSA controller.

(2) Active Secondary Users (ASUs): once the RSUs obtain spectrum resources (allocated resource blocks) from the LSA controller, they should deliver a compensation fee based on the requested resources. Then, the LSA licensee will lease the underutilized LSA spectrum resources to the RSUs. After the leasing procedure is finished successfully, the RSUs formally change their status to ASUs.

(3) Finished ASUs (FASUs): at each time instant $t_{i}$, some of the ASUs finish their operations and release the allocated spectrum resources back to the incumbent.

(4) Rejected ASUs (RASUs): in case the incumbent requests the leased spectrum resources back, the ASUs are forced to vacate the cellular band immediately, even if their active transmission is not finished.

(iii) Regulator: it is the entity responsible for granting permissions over the incumbent's cellular band. It harmonizes spectrum usage and opens a path to spectrum optimization via controlled sharing, which is the key responsibility of the National Regulatory Authority (NRA).

3.2. Utilized Attributes. The main utilized attributes in the considered system model are linked with RSUs and ASUs, since those two states of the SUs in the leasing process are crucial for our CBRM framework. The attributes of RSUs include (i) requested bit rate (BR) and (ii) rejected history $(\mathrm{RH})$. The main real-time attributes for the ASUs are listed as follows:

(i) Received Signal Strength Indicator (RSSI): an indication of the power level being received by the ASUs

(ii) Usage pattern (AvrST): average service time calculated by utilizing the ASU's service started time (SST) and the current time instant $t_{i}$

(iii) Activity factor (DUR): the average download/upload ratio of each ASU, calculated from the ASU's service started time up to the current time instant

(iv) Mobility pattern (MP): movement direction of each ASU within the cell coverage where "1" represents direction towards the eNodeB; " 2 " stands for the opposite direction [30].

The SUs send a request as RSUs; $L_{\mathrm{RSU}}\left(t_{i}\right)$ is further updated according to the following:

$$
\begin{aligned}
& L_{\mathrm{RSU}}\left(t_{i}\right) \\
& \quad=\left\{\mathrm{RSU}_{j}=\left(\mathrm{BR}_{j}, \mathrm{RH}_{j}\right) \mid 1 \leq j \leq \alpha\left(t_{i}\right), 1 \leq i \leq T\right\},
\end{aligned}
$$

where $T$ is the total time interval, $t_{i}$ represents the $i$ th time instant, and $\alpha\left(t_{i}\right)$ shows the number of RSUs modeled as a
Poisson distribution at time instant $t_{i}$, that is, $\mathrm{RSU}_{\text {ShowRate }}$. The list of ASUs, $L_{\mathrm{ASU}}\left(t_{i}\right)$, is calculated as follows:

$$
\begin{gathered}
L_{\mathrm{ASU}}\left(t_{i}\right)=\left\{\mathrm{ASU}_{j}|0 \leq j \leq| L_{\mathrm{ASU}}\left(t_{i-1}\right)|-| L_{\mathrm{FASU}}\left(t_{i}\right) \mid\right. \\
\left.-\left|L_{\mathrm{RASU}}\left(t_{i}\right)\right|+\left|L_{\mathrm{ASU}}\left(t_{i}\right)\right|, \quad 1 \leq i \leq T\right\} .
\end{gathered}
$$

The number of FASUs in $L_{\mathrm{FASU}}\left(t_{i}\right)$ is assumed to be upper-bounded by the number of ASUs from preceding time interval. Next, the list of FASUs is derived as follows:

$$
\begin{aligned}
& L_{\mathrm{FASU}}\left(t_{i}\right) \\
& \quad=\left\{\text { FASU }_{j}|0 \leq j \leq| L_{\mathrm{ASU}}\left(t_{i-1}\right) \mid, \quad 1 \leq i \leq T\right\} .
\end{aligned}
$$

Further, the number of RASUs in $L_{\text {RASU }}\left(t_{i}\right)$ is also upperbounded by the number of sensed PUs in the current time interval $t_{i}$ that exceeds the number of $\operatorname{URB}\left(t_{i}\right)$ :

$$
\begin{aligned}
& L_{\mathrm{RASU}}\left(t_{i}\right)=\left\{\mathrm{RASU}_{j}|0 \leq j \leq| L_{\mathrm{PU}}\left(t_{i}\right) \mid\right. \\
& \left.-\operatorname{URB}\left(t_{i}\right), 1 \leq i \leq T\right\}
\end{aligned}
$$

where $\operatorname{URB}\left(t_{i}\right)$ stands for the number of underutilized blocks (URBs) and $L_{\mathrm{PU}}\left(t_{i}\right)$ is the list of PUs derived from [29]. It is assumed that a PU is characterized by an on-rate, which is denoted by $\beta$. It is the probability of PU's activity per time interval $\left(\mathrm{PU}_{\mathrm{UsageRate}}\right)$. In [31], it is stated that the parameter $\beta$ varies between $15 \%$ and $85 \%$. In our scenario, we consider that $\beta$ follows a binomial distribution and $\beta\left(t_{i}\right)$ denotes the on-rate of a PU at time instant $t_{i}$ with $0 \leq \beta\left(t_{i}\right) \leq 1$. At each time interval, the size of $L_{\mathrm{PU}}\left(t_{i+1}\right)$ is updated with respect to the total number of resource blocks $\varphi$ and $\beta\left(t_{i}\right)$ as follows:

$$
L_{\mathrm{PU}}\left(t_{i}\right)=\varphi \times \beta\left(t_{i}\right) .
$$

Following the above, the total number of RSUs, ASUs, FASUs, and RASUs can be calculated as follows:

$$
L_{x \mathrm{SU}}\left(t_{i}\right)=\left\{x \mathrm{SU}_{m} \mid 0 \leq m \leq \phi, 1 \leq i \leq T\right\},
$$

where $T$ is the total time interval and $t_{i}$ represents the $i$ th time instant and $\phi$ corresponds to the total number of $x \mathrm{SUs}$, by adding the number of RSUs, ASUs, FASUs, and RASUs from the related list at $t_{i}$ as follows:

$$
\begin{aligned}
\phi= & \left|L_{\mathrm{RSU}}\left(t_{i}\right)\right|+\left|L_{\mathrm{ASU}}\left(t_{i}\right)\right|+\left|L_{\mathrm{FASU}}\left(t_{i}\right)\right| \\
& +\left|L_{\mathrm{RASU}}\left(t_{i}\right)\right| .
\end{aligned}
$$

For the sake of completeness, we assume allocation of exactly one resource block to one user at a given time instant $t_{i}$.

Finally, after the URB calculation at a given time instant $t_{i}$, four main use-cases can be introduced [31]:

(i) Case 1: $\left|L_{\mathrm{RSU}}\left(t_{i}\right)\right| \leq \operatorname{URB}\left(t_{i}\right)$.

(ii) Case 2: $0 \leq \operatorname{URB}\left(t_{i}\right)<\left|L_{\mathrm{RSU}}\left(t_{i}\right)\right|$.

(iii) Case 3: $0 \leq \mathrm{URB}\left(t_{i}\right)<\left|L_{\mathrm{PU}}\left(t_{i}\right)\right|$.

(iv) Case 4: $\left|L_{\mathrm{PU}}\left(t_{i}\right)\right|=\varphi$. 
TABLE 1: Pairwise comparison matrix for ASU attributes.

\begin{tabular}{lcccc}
\hline User attributes & RSSI & AvrST & DUR & MP \\
\hline RSSI & 1 & $1 / \Theta_{21}$ & $1 / \Theta_{31}$ & $1 / \Theta_{41}$ \\
AvrST & $\Theta_{21}$ & 1 & $1 / \Theta_{32}$ & $1 / \Theta_{42}$ \\
DUR & $\Theta_{31}$ & $\Theta_{32}$ & 1 & $1 / \Theta_{43}$ \\
MP & $\Theta_{41}$ & $\Theta_{42}$ & $\Theta_{43}$ & 1 \\
\hline
\end{tabular}

3.3. Criteria-Based Rejection Model. The proposed CBRM framework targets the third use-case mentioned in the previous text, that is, $0 \leq \mathrm{URB}\left(t_{i}\right)<\left|L_{\mathrm{PU}}\left(t_{i}\right)\right|$. Following this condition, the LSA licensee must vacate the leased spectrum resources, since the LSA controller has to maintain the target QoS and QoE parameters for the incumbents (PUs).

Generally, the probability of ASU rejection increases during peak hours. In this work, the rejection functionality is modeled for two scenarios: (i) increasing ASU's target satisfaction, while selecting the appropriate ASUs to be rejected, and (ii) increasing the network revenue provided to RASUs, paying the so-called CC, which can be used during further attempts to allocate the underutilized spectrum resources, that is, prioritization of earlier rejected ASUs. To this end, the function Reject $\left(L_{\mathrm{ASUs}}\left(t_{i}\right)\right)$; DecisionBasedRejectionPolicy represents the generalized form of rejection process mentioned as the third use-case above. As an output, it returns $L_{\mathrm{RASU}}\left(t_{i}\right)$. DecisionBasedRejectionPolicy is composed of the following steps:

(1) Identifying the decision criterion for SUs and their priority

(2) Normalization process of decision criterion

(3) Importance degree allocation (priority vector)

(4) Evaluating the decision made

(5) Selection of the appropriate ASUs that will be rejected (as stated in (10)).

The principles of DecisionBasedRejectionPolicy algorithm, proposed in this work, are detailed in Algorithm 1. At the beginning of the decision process, the MNO collects all of the input decision criteria (i.e., RSSI, AvrtST, DUR, and MP) from $L_{\mathrm{ASU}}$. The obtained value for each ASU is normalized based on the cost/benefit type of a particular criterion (see line (1) in Algorithm 1) [32]. Therefore, each criterion of ASUs has to be compared with the criteria of other ASUs, considering the list of criteria for ASUs. This comparison leads to stability among ASUs, which is the crucial step in the process of finding a rejection ratio (RR) for each and every ASU.

Further, a numerical weight (priority) is computed for each criterion of the ASUs by the LSA licensee; see line (7) in Algorithm 1. The PriorityVector shows the importance degree of each criterion as compared to other criteria, where $\sum_{j=1}^{\mid \text {Criterion| }}$ PriorityVector $(j)=1$. The decision on the priority assignment, from the LSA licensee side, can be adaptive at each $t_{i}$ according to the peak and the off-peak hours. In this work, we have implemented the Analytic Hierarchy Process (AHP) at the side of the LSA licensee. AHP is one of the multicriteria decision-making methods that was considered in [33-35].

Developing a single pairwise comparison matrix for the criteria is the first step when the AHP is utilized. Table 1 shows the pairwise comparison matrix composed of four ASU's attributes mentioned in detail within Section 3.2.

The value $\Theta_{i j}$ represents a set with respect to the scale of absolute judgment in AHP method [22,32,34]. As commonly used in AHP, the values $\Theta_{i j}$ vary between 1 and 9. The reciprocal relationship between the $i$ th and $j$ th attributes is given by $\Theta_{i j}=1 / \Theta_{i j}$.

Next, the $n$th root-of-product values in each row is calculated. The Consistency Ratio (CR) is further derived as shown in (8), where consistency of the pairwise comparison from the LSA licensee side is provided.

$$
\mathrm{CR}=\frac{\text { Consistency Index }(\mathrm{CI})}{\text { Random Index }(\mathrm{RI})} .
$$

The Consistency Index (CI) is produced as

$$
\mathrm{CI}=\frac{\left(\lambda_{\max }-n\right)}{(n-1)},
$$

where $n$ is the number of compared criterion. In the described use-case, four different criteria are compared. Further, $\lambda_{\max }$ shows the sum of the priority vector values, as mentioned at Step (8) in Algorithm 1.

Continuing our description of the proposed CBRM framework, the RI stands for the direct function of the number of criteria taken into consideration [33]. Finally, the CR (see (8)) provides the decision-maker with the information on how consistent was the process of pairwise comparison. After the CR is calculated, two main (and different) conditions are to be checked:

(i) $\mathrm{CR}<0.10$ : pairwise comparisons made by the MNOs were found to be relatively consistent

(ii) $\mathrm{CR}>$ 0.10: reevaluation of the completed pairwise comparison processes should be taken into account by the MNOs.

When the condition is reached, the MNO can make its decision and choose the appropriate ASUs; hence, we have the following:

$$
\operatorname{RR}_{\mathrm{ASU}}(i)=\sum_{i=1}^{\left|L_{\mathrm{ASU}}\left(t_{i}\right)\right| \mid \text { criterion } \mid} \sum_{j=1}^{r} r(i, j) \times \text { Priority Vector }_{j},
$$




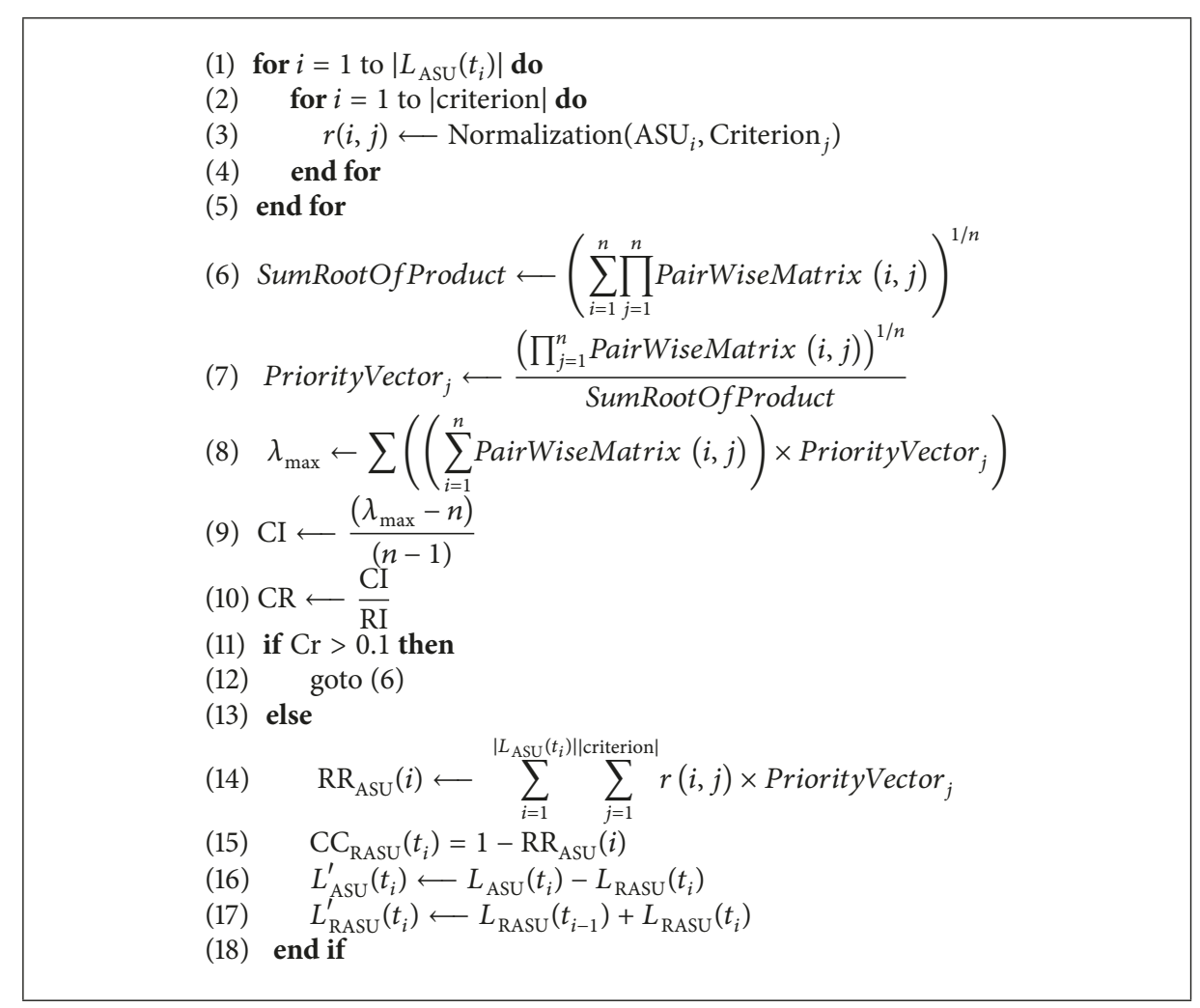

Algorithm 1: DecisionBasedRejectionPolicy algorithm.

where |criterion| is considered as a list of 4 parameters mentioned in Section 3.2. The ASUs will be rejected according to the increasing order of $\mathrm{RR}_{\mathrm{ASU}}\left(t_{i}\right)$, where the number of RASUs can be found as follows:

$$
\left|L_{\mathrm{RASU}}(i)\right|=\left|L_{\mathrm{ASU}}\left(t_{i}\right)\right|-\left(\varphi-\left|L_{\mathrm{PU}}\left(t_{i}\right)\right|\right) \text {. }
$$

If every RASU is willing to request new spectrum resources in the future as the $\mathrm{RSU}$, the CC ratio will be applied to the price function as CCi in (13) and these RSUs will pay a lower price than other RSUs based on the CC ratio. This behavior is connected with user satisfaction in both (i) rejected and (ii) spectrum requesting steps. The CC ratio is calculated as follows:

$$
\mathrm{CC}_{\mathrm{RASU}}\left(t_{i}\right)=1-\mathrm{RR}_{\mathrm{ASU}}\left(t_{i}\right) .
$$

After assigning the calculated CC ratio to RASUs at the time instant $t_{i}$, it should be (i) removed from the ASUs list and (ii) added to the existing RASUs list. Further, the lists of ASUs and RASUs should be updated.

Recalling Section 3, RSUs will have an opportunity to lease underutilized spectrum resources (URBs) if and only if they pay the requested resource block's fee. The goal of this approach is to obtain higher network revenue, while motivating users to request the underutilized spectrum more frequently. The constructed pricing function offers an opportunity for the LSA licensee(s) to reach the mentioned goal. Therefore, at each time instant $t_{i}$ (after the RSUs receive an approval to access the under-utilized spectrum), the RSUs will be charged for the resource allocation. The price paid by the RSUs can be measured in monetary units or alternatively it can be measured in virtual currency [27]. The pricing function is further calculated as follows:

$$
\text { Price }_{\mathrm{RSU}}\left(t_{i}\right)=P_{i} \times\left(1-\mathrm{CC}_{\mathrm{RSU}}\right),
$$

where $P_{i}$ is the price paid by the RSUs and CC stands for the compensation cost in (12). For the investigated pricing function, there are two important cases:

(i) If $\mathrm{CC}_{\mathrm{RSU}}==0$ then Price RSU $_{i}\left(t_{i}\right)=p_{i}$.

(ii) If $\mathrm{CC}_{\mathrm{RSU}}==1$ then Price $_{\mathrm{RSU}}\left(t_{i}\right)=0$.

The first case takes place for the RSUs without the rejected history in the network up to the current time instant. Therefore, the default price will be set for the RSU(s). The second case refers to the situation where the RSU had $100 \%$ rejection history in the cellular network. Even though the second case decreases the network revenue at the time instant $t_{i}$, in the long-term perspective it can be expected to observe repeated attempts from the RSUs to use the underutilized spectrum. Finally, Figure 3 summarizes the decision flow of the CBRM framework at time instant $t_{i}$. For the sake of completeness, a summary of the variables used in our system model is given in "List of Used Variables" in Nomenclature. 


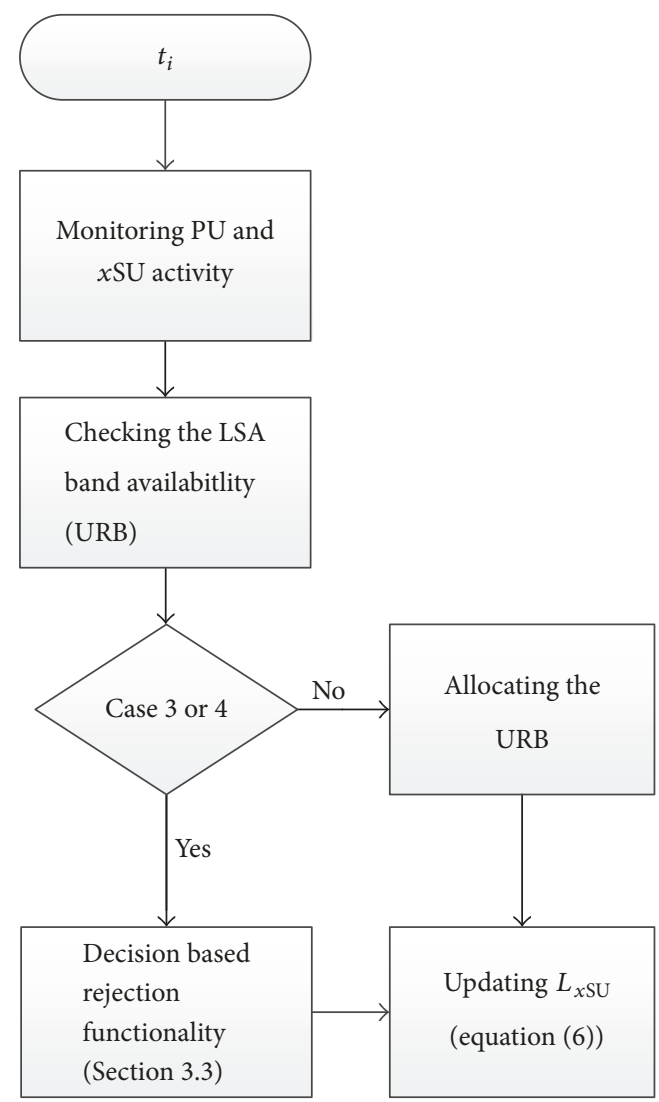

FIgURE 3: Decision flow diagram of the system model at $t_{i}$.

TABLE 2: Simulation parameters.

\begin{tabular}{lc}
\hline Parameter & Value \\
\hline Simulation time & $100 \mathrm{~s}$ \\
Number of resource blocks & $25 \mathrm{RB}$ \\
$\mathrm{PU}_{\text {UsageRate }}$ & {$[0.25 ; 0.75] \%$} \\
$\mathrm{RSU}_{\text {ShowRate }}$ & {$[0.25 ; 0.75] \%$} \\
$\mathrm{RSSI}$ & {$[-100 ;-30] \mathrm{dBm}$} \\
Mobility pattern & {$[1 ; 2]$} \\
Downlink channel bit rate & {$[1 ; 33] \mathrm{Mbps}$} \\
Uplink/downlink ratio & {$[0.01 ; 1]$} \\
\hline
\end{tabular}

\section{Simulation Results}

To adequately evaluate the proposed system model formulated in Section 3, we decided to utilize as our simulation parameters the data obtained from the experimental 3GPP LTE-A system located at the Department of Telecommunications, Brno University of Technology (BUT), Czech Republic. It supports functionality of LTE Release 10 communications system. The list of employed simulation parameters in the developed framework is shown in Table 2.

In this work, we assume $\mathrm{PU}_{\text {UsageRate }}=0.25$ and $\mathrm{RSU}_{\text {ShowRate }}=0.25$ for the off-peak hours. Further, $\mathrm{PU}_{\text {UsageRate }}$ and $\mathrm{RSU}_{\text {ShowRate }}$ are set to 0.75 represent the peakhour parameters. According to the LTE-A system design, the RSSI is configured in the range $[-100 ;-30] \mathrm{dBm}$ and the
TABLE 3: Simulation results for off-peak and peak hours.

\begin{tabular}{lcc}
\hline \multirow{2}{*}{ Parameter } & \multicolumn{2}{c}{ Values for off-peak and peak hour } \\
& Off-peak & Peak \\
\hline Total number of RSUs & 622 & 1878 \\
Total number of ASUs & $357(57.39 \%)$ & $166(8.86 \%)$ \\
Total number of FASUs & $321(89.91 \%)$ & $76(45.78 \%)$ \\
Total number of RASUs & $17(4.76 \%)$ & $82(49.39 \%)$ \\
Number of remaining ASUs & $19(5.32 \%)$ & $8(4.82 \%)$ \\
Total network revenue & 147603 & 78532 \\
\hline
\end{tabular}

BR for the downlink channel is set in the range $[1 ; 33] \mathrm{Mbit} / \mathrm{s}$. The mobility pattern is denoted as " 1 " and " 2 ," where " 1 " represents the direction towards the eNodeB; " 2 " stands for the opposite direction. The total number of usable resource blocks in the system is considered to be 25 (since the available system bandwidth is $5 \mathrm{MHz}$ ) for all time intervals. The simulation constructed in Matlab environment is set to 100 time intervals with a certain time duration. The base price is set to $p=500$.

In this modeled scenario, the URB, PUs, and ASUs are involved in the networking. As shown in Figure 4(a), most of the RSUs are going to be changed to ASUs. However, there are still URBs at specific time instants. During the peak hours (see Figure $4(\mathrm{~b})$ ), almost $75 \%$ of the resource blocks belong to the PUs. In this case, not only is the limited number of URBs available but also the RASUs are encountered. In more detail, PUs and RSUs exist in the network at the same time, which is based on $\mathrm{PU}_{\text {UsageRate }}$ and $\mathrm{RSU}_{\text {ShowRate }}$; this behavior is according to the mentioned use-cases; see Section 3.2.

As simulation time progresses, the ASUs $\left(L_{\mathrm{ASU}}\right)$ and the status of each of them will be changed to FASU, RASU, or there will still be ASUs at each time instant; for example, some of the ASUs will leave the mobile network as FASUs and some users may be rejected (RASU) according to case 3: $0 \leq \mathrm{URB}\left(t_{i}\right)<\left|L_{\mathrm{PU}}\left(t_{i}\right)\right|$; see Section 3.2.

The important simulation results are detailed in Table 3 for the off-peak and peak hours, respectively. The Number of Remaining ASUs is further calculated as follows:

$$
\mathrm{NR}_{\mathrm{ASUs}}=L_{\mathrm{ASU}}-L_{\mathrm{FASU}}-L_{\mathrm{RASU}} .
$$

The number of RSUs during the off-peak hours (622) is lower than that over the peak hours (1878). Further, $57.39 \%$ of the RSUs have become ASUs during the off-peak hours and about $8.87 \%$ did that during the peak hours. This behavior is due to the fact that the LTE network operates with $25 \mathrm{RB}$ (5 MHz bandwidth). Further, the number of RASUs during the peak hours (49.39\%) reflects the Total Network Revenue (TNR), which is lower during the off-peak time. However, over the off-peak hours, $89.91 \%$ of the ASUs finished their data transmission successfully (FASUs $=21$ ) and $4.76 \%$ of ASUs are rejected (RASUs) in total.

Table 3 shows the total number of RASUs (17) during the off-peak hours and the peak hours (82). Figure 5 reports the total number of time intervals when the RASUs were activated in the network, see Figure 5(a), and the estimated price 


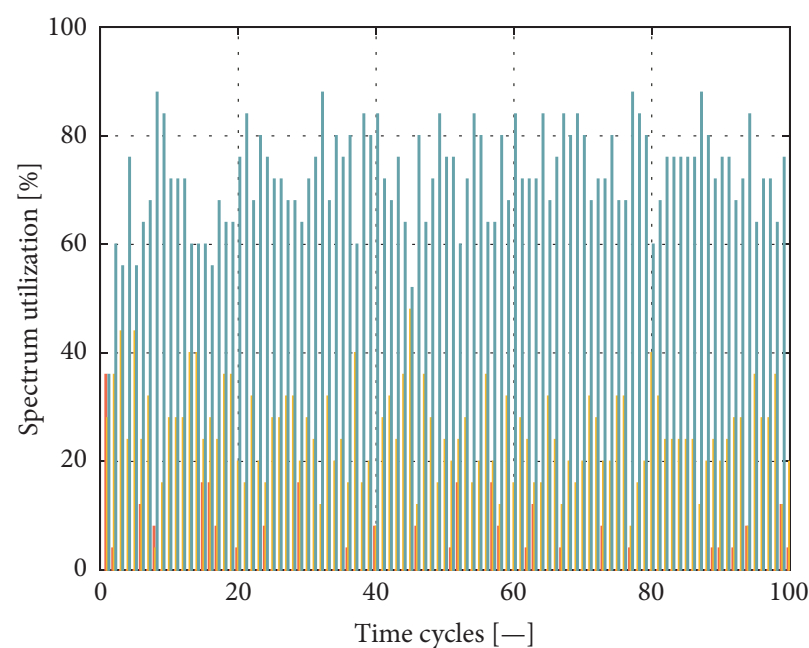

URB

PUs ASUs

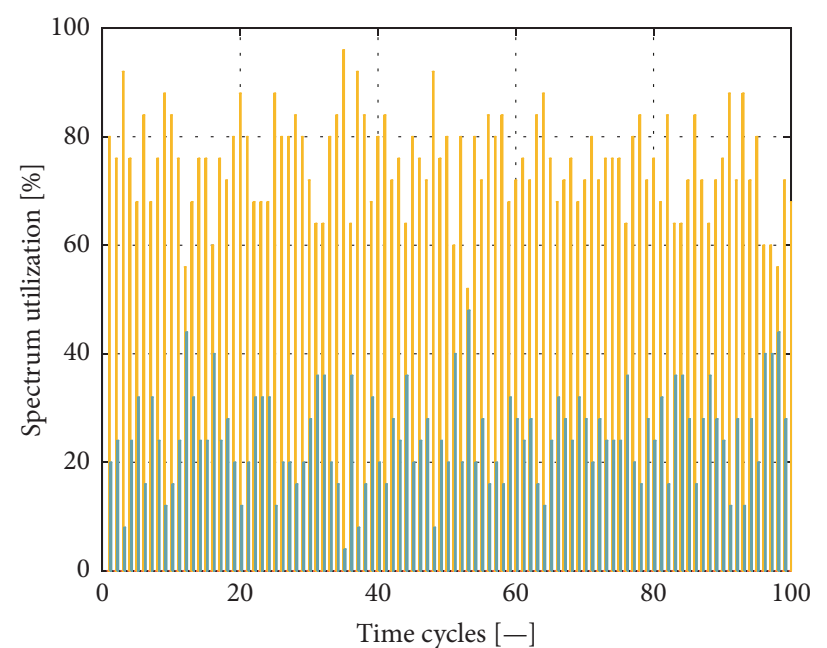

URB

PUs

ASUs

(a)

(b)

FIGURE 4: Spectrum utilization during off-peak (a) and peak (b) hours.

of their next connection attempt (Figure 5(b)) during the off-peak hours. This reflects the fact that whenever the RASUs are willing to request service time, for example, a connection to the mobile network, the payment will be as shown in Figure 5(b) for each of them.

Further, Figure 6 details the situation for the peak hours: as can be seen, if the only criterion for the rejected ASUs was the AvrST, there should be agreement between (i) Figures 5(a) and 5(b), as well as (ii) Figures 6(a) and 6(b). However, the obtained results vary even for the ASUs with the same AvrST. For example, AvrST ( $\left.\mathrm{ASU}_{6}\right)$ and AvrST $\left(\mathrm{ASU}_{7}\right)$ are set to 9 time intervals. On the other hand, the estimated price for the selected ASUs differs $\left(\mathrm{ASU}_{6}=334.083 ; \mathrm{ASU}_{7}=\right.$ 320.629). This situation clearly indicates the importance of another criterion for the CC calculation in the proposed CBRM model. We proceed with considering the said criterion in the following section.

\section{User Selection Methodology}

In this section, we continue our description of the constructed model (formulated in the previous Section 4). We focus on selecting the appropriate RASUs among the ASUs. Step-by-step selection functionality of the served users utilizes two key principles: (i) availability of the LSA band and (ii) decision-based rejection functionality as detailed in Figure 7. In order to study performance of the DecisionBasedRejectionPolicy algorithm, the second time interval $\left(t_{2}\right)$ is captured. At the beginning, the LSA licensee checks whether the spectrum resources are available at $t_{2}$. According to the $\mathrm{URB}\left(t_{2}\right)$ and $\left|L_{\mathrm{PU}}\left(t_{2}\right)\right|$, the number of RASUs is $\left|L_{\mathrm{RASU}}\left(t_{2}\right)\right|=$ 2; see also the condition given in case 3: $0 \leq \operatorname{URB}\left(t_{i}\right)<$ $\left|L_{\mathrm{PU}}\left(t_{i}\right)\right|$; see Section 3.2.
The following step in our consideration of the ASU's criteria and the importance degree calculation is to employ the AHP: the LSA licensee considers all 6 criteria. The corresponding importance degrees are shown in Table 4. The LSA licensee calculates the RR based on the condition of ASUs at the time instant $t_{2}$, with the corresponding parameters shown in Table 5. The AvrST is the most important attribute for both sides (the LSA licensee and the incumbent (PU)). The order of the remaining attributes (criteria) is the following: (i) RSSI, (ii) rejected history (RH), (iii) mobility pattern, (iv) bit rate (BR), and (v) download/upload rate.

As it was discussed in Section 4, a comparison between the ASUs based only on the AvrST attribute is not feasible (see Table 5, where the AvrST attribute is set to 1 for all the ASUs). To this end, the second attribute (RSSI) was added to our consideration. In this case, two ASUs nominated for rejection will be $\mathrm{ASU}_{\mathrm{ID}=3}$ and $\mathrm{ASU}_{\mathrm{ID}=5}$ with RSSI $=-78 \mathrm{dBm}$ and RSSI $=-82 \mathrm{dBm}$, respectively. Another important criterion is $\mathrm{RH}$, where $\mathrm{ASU}_{\mathrm{ID}=1}$ and $\mathrm{ASU}_{\mathrm{ID}=4}$ are nominated to be rejected, since they have the lowest rejection ratio. Considering the MP, the ASUs selected for rejection will be $A S U_{I D=1}$ and $\mathrm{ASU}_{\mathrm{ID}=2}$. Further, the $\mathrm{BR}$ attribute is considered and, by following this criterion, $\mathrm{ASU}_{\mathrm{ID}=1}$ and $\mathrm{ASU}_{\mathrm{ID}=5}$ are the candidates for rejection. Finally, when DUR is taken into account, $\mathrm{ASU}_{\mathrm{ID}=4}$ and $\mathrm{AS} \mathrm{U}_{\mathrm{ID}=5}$ are going to be rejected, since they have the maximum download/upload ratio at a given time instant $t_{2}$.

Continuing the user selection process, Table 6 provides information about the RR, rejected order, and CC of the ASUs. The LSA licensee selects two ASUs for rejection according to $\left|L_{\mathrm{RASU}}\left(t_{2}\right)\right| \rightarrow \mathrm{ASU}_{\mathrm{ID}=1}$ and $\mathrm{ASU}_{\mathrm{ID}=5}$, which have the lowest CC based on (12) (CC ASU $U_{\mathrm{ID}=1}=0.1392$; $\mathrm{CC} \mathrm{ASU} \mathrm{ID}=5=$ 0.1429). Further, if the LSA licensee rejected $A S U_{I D=4}$ (third ASU selected for rejection, see Table 5), the LSA licensee must 
TABLE 4: Importance degree of each criterion of the DecisionBasedRejecti onPolicy based on AHP method.

\begin{tabular}{lcr}
\hline Criteria & & AHP \\
BR & Importance order (MNO view) & Importance degree (AHP view) \\
RH & 5 & 0.0573 \\
RSSI & 3 & 0.2005 \\
AvrST & 2 & 0.2526 \\
DUR & 1 & 0.3460 \\
MP & 6 & 0.0422 \\
\hline
\end{tabular}

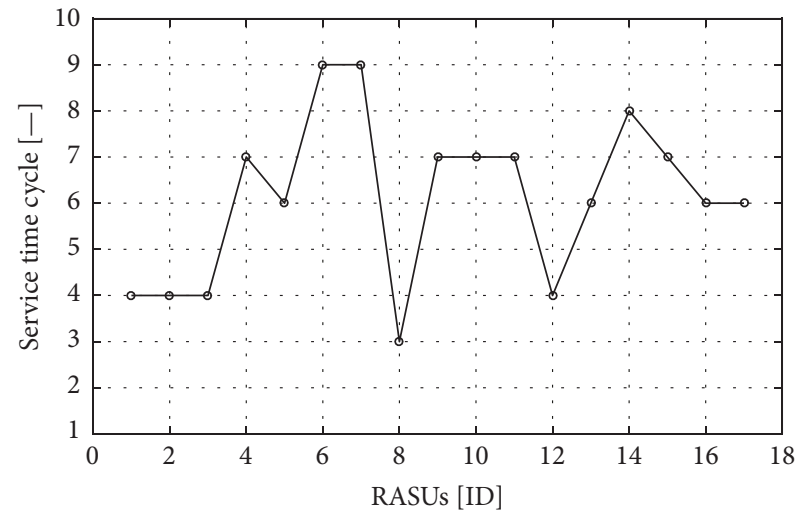

(a)

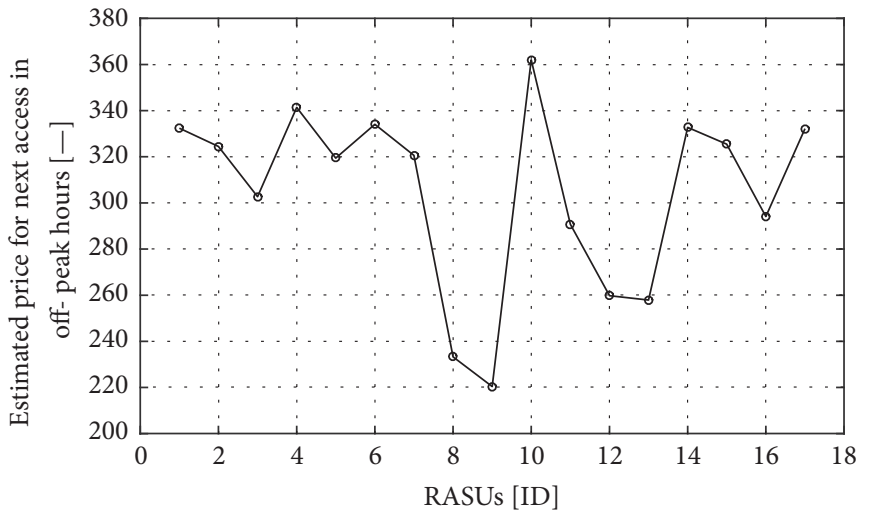

(b)

FIgURE 5: Number of time intervals that RASUs are active (a) and estimated price for their next connection (b) during off-peak hours.

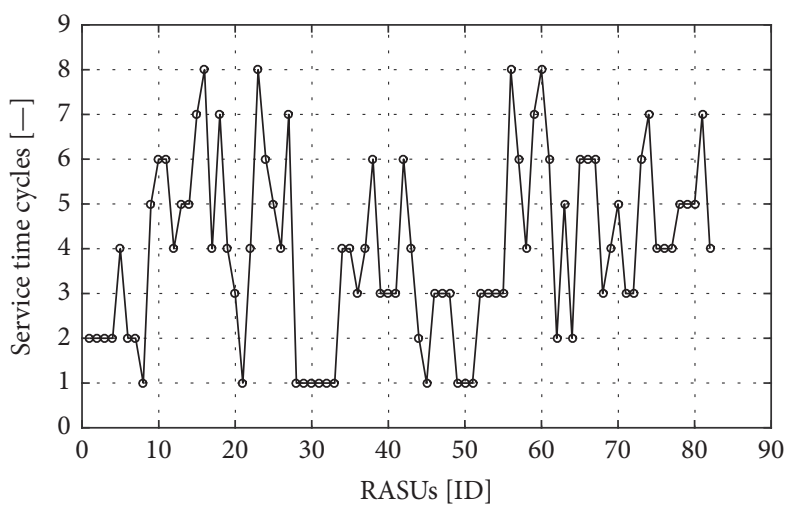

(a)

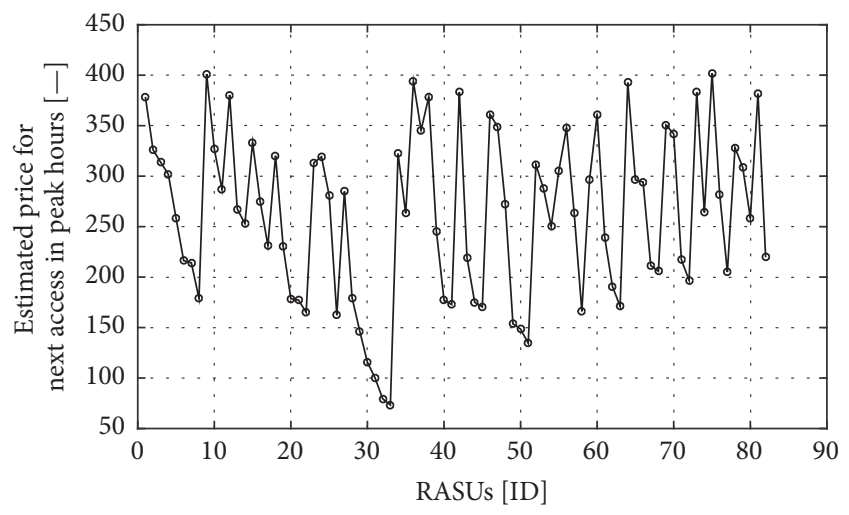

(b)

FIGURE 6: Number of time intervals that RASUs are active (a) and estimated price for their next connection (b) during peak hours.

charge this ASU for 336.80. Since the base price is assumed to be $p=500, \mathrm{ASU}_{\mathrm{ID}=1}$ and $\mathrm{ASU}_{\mathrm{ID}=5}$ will charge 430.40 and 428.55 for the spectrum resources, respectively. This consideration keeps $\mathrm{ASU}_{\mathrm{ID}=4}$ more suitable for the LSA licensee (i.e., increasing the network revenue). On the other hand, this decision will keep $A S U_{I D=4}$ satisfied as its DUR and BR are lower than those for other two rejected ASUs. Further, a variable spectrum resource price (Price ${ }_{\mathrm{RSU}}$ ) in Table 5 confirms that the price paid for using the LSA band by every accepted RSU depends on their rejection history $(\mathrm{RH})$ that is calculated across previous time intervals. As an output of the decision process, DecisionBasedRejectionPolicy algorithm has the potential to balance all the probabilities and maximize the network performance together with the user satisfaction.

\section{Conclusion}

A Criteria-Based Resource Management framework for the MNO LSA-based operation was proposed in this work. The presented approach is built on the MNO's decisions regarding both pricing and rejection policies with several types of users, the corresponding attributes, and a number of the MNO decision scenarios. The constructed system model illustrates the MNO decisions across several situations in terms of 
TABLE 5: Parameters of ASUs at 2nd time instant (status table).

\begin{tabular}{|c|c|c|c|c|c|c|}
\hline \multirow{2}{*}{$\begin{array}{l}\text { Parameter name } \\
\mathrm{ASU}_{\mathrm{ID}}\end{array}$} & \multicolumn{6}{|c|}{ StatusTable $_{\text {ASU }}$ in 2nd time instant } \\
\hline & 1 & 2 & 3 & 4 & 5 & 6 \\
\hline Price $_{\mathrm{RSU}}$ & 468.5535 & 380.5031 & 402.5157 & 474.8428 & 427.673 & 391.5094 \\
\hline RSSI $[\mathrm{dBm}]$ & -66 & -71 & -78 & -31 & -82 & -60 \\
\hline AvrST & 1 & 1 & 1 & 1 & 1 & 1 \\
\hline DUR & 36 & 32 & 1 & 67 & 89 & 43 \\
\hline MP & 2 & 2 & 1 & 1 & 1 & 1 \\
\hline $\mathrm{BR}[\mathrm{Mbit} / \mathrm{s}]$ & 33 & 6 & 17 & 21 & 27 & 14 \\
\hline $\mathrm{RH}[-]$ & 0.2 & 0.76 & 0.62 & 0.16 & 0.46 & 0.69 \\
\hline
\end{tabular}

TABLE 6: Parameters of ASUs at 2nd time instant (decision made based on DecisionBasedRejectionPolicy).

\begin{tabular}{lccccc}
\hline Parameter name & \multicolumn{5}{c}{ DecisionBasedRejectionPolicy results at 2nd time instant } \\
\hline Rejection_Ratio (RR) & 0.86084 & 0.640409 & 0.645008 & 0.673629 & 0.857106 \\
Rejected order & 1 & 5 & 4 & 3 & 0.601851 \\
CC & 0.1392 & 0.3596 & 3550 & 0.3264 & 0 \\
\hline
\end{tabular}

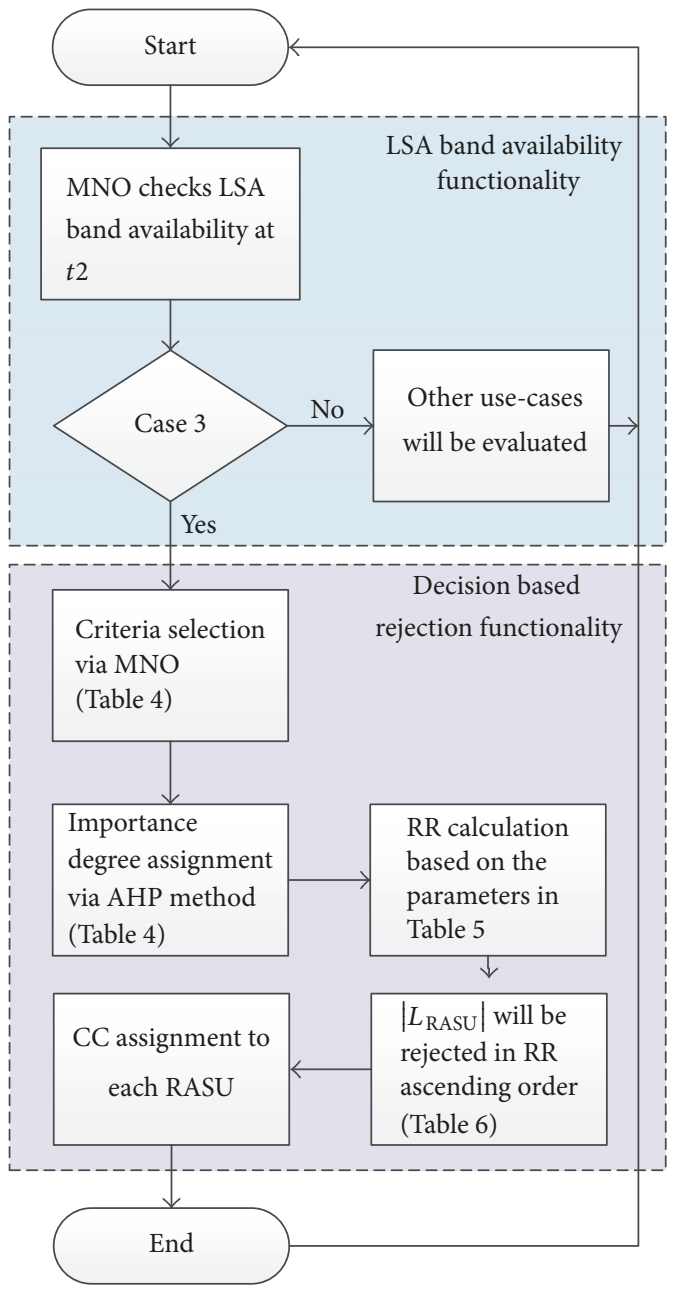

FIGURE 7: Selection functionality for served users.

rejection of the appropriate ASUs, in order to balance the user and the MNO satisfaction in both the pricing and rejection processes.

In this work, the rejection functionality is modeled for two scenarios (i) increasing ASU's target satisfaction, while selecting the appropriate ASUs to be rejected via RR, and (ii) increasing the network revenue provided to RASUs, paying the CC, which can be used during further attempts to allocate the underutilized spectrum resources, that is, prioritization of earlier rejected ASUs. On the other hand, the price paid by RSUs for the allocated spectrum resources depends on their rejection history.

A question that emerged during the construction of this methodology is how to analyze a number of RSUs that the MNO will not charge (Price $\operatorname{RsU}\left(t_{j}\right)=0$ ) regarding their $\mathrm{RH}$ $\left(\mathrm{CC}_{\mathrm{RSU}}=1\right)$. Granted support from the operators, there is a possibility of reaching the desired trade-off during peak/offpeak hours and achieving the corresponding effect on the Total Network Revenue, which can be evaluated via different decision-making criteria and the importance degree based on the AHP at the side of the LSA licensee.

The simulation results were also obtained based on our investigations of the CBRM framework, which has been specifically designed to provide insights into dynamic LSA management in 5G mobile networks. Our findings support the key assumptions of the created system model and show in detail the spectrum utilization as well as how the proposed method can motivate the rejected users to request the service from the same network in the future. As an output of the decision process, CBRM can balance all the rejection and requesting probabilities as well as maximize the network performance together with the user satisfaction or QoE.

\section{Nomenclature}

List of Frequently Used Acronyms

AHP: Analytic Hierarchy Process

ASU: Active Secondary User

CBRM: Criteria-Based Resource

Management

CI: $\quad$ Consistency Index

CR: Consistency Ratio

CRN: Cognitive Radio Network

FASU: Finished Active Secondary User 
MNO: Mobile Network Operator

NRA: National Regulatory Authority

PU: Primary User

RASU: Rejected Active Secondary User

RI: $\quad$ Random Index

RSU: Requesting Secondary User

SU: Secondary User

URB: Underutilized Resource Block

LSA: Licensed Shared Access.

\section{List of Used Variables}

$\begin{array}{ll}T: & \text { Total time interval } \\ t_{i}: & \text { Time instant } \\ \varphi: & \text { Total number of resource blocks } \\ \phi: & \text { Total number of RSUs, ASUs, FASUs, } \\ & \text { and RASUs at } t_{i} \\ \mathrm{SB}: & \text { Spectrum band } \\ \mathrm{RB}: & \text { Requested bit rate } \\ \mathrm{RH}: & \text { Reject history } \\ \mathrm{DUR}: & \text { Download/upload ratio } \\ \mathrm{MP}: & \text { Mobility pattern } \\ \text { AvrST: } & \text { Average service time } \\ \alpha\left(t_{i}\right): & \text { The number of RSUs modeled as a } \\ \beta\left(t_{i}\right): & \text { Poisson distribution at } t_{i} \\ \lambda_{\text {max }}: & \text { On-rate of a PU at } t_{i} \\ n: & \text { Sum of the priority vector values } \\ & \text { Number of compared criteria } \\ \text { Price } & \text { (|criterion| }) \\ P_{i}: & \text { Calculated price for the future } \\ L_{\mathrm{PU}}\left(t_{i}\right): & \text { requests by RSU } \\ L_{\mathrm{RSU}}\left(t_{i}\right): & \text { List of PUs at } t_{i} \\ L_{\mathrm{ASU}}\left(t_{i}\right): & \text { List of } \text { ASUs at } t_{i} \\ L_{\mathrm{FASU}}\left(t_{i}\right): & \text { List of FASUs at } t_{i} \\ L_{\mathrm{RASU}}\left(t_{i}\right): & \text { List of RASUs at } t_{i} \\ \mathrm{CC}: & \text { Compensation cost } \\ \mathrm{RR}: & \text { Reject ratio. }\end{array}$

\section{Conflicts of Interest}

The authors declare that there are no conflicts of interest.

\section{Acknowledgments}

The described research was supported by the National Sustainability Program under Grant LO1401. For the research, infrastructure of the SIX Center was used. The publication has been prepared with the support of the "RUDN University Program 5-100" and funded by RFBR according to the Research Projects no. 16-07-00766 and no. 17-07-00845.

\section{References}

[1] "Cisco Visual Networking Index: Global Mobile Data Traffic Forecast Update, 2016-2021 White Paper," March 2017, https:// www.cisco.com/c/en/us/solutions/collateral/service-provider/ visual-networking-index-vni/mobile-white-paper-c1l-520862 .html.
[2] M. A. Clark and K. Psounis, "Equal Interference Power Allocation for Efficient Shared Spectrum Resource Scheduling," IEEE Transactions on Wireless Communications, vol. 16, no. 1, pp. 5872, 2017.

[3] V. Sridhar, T. Casey, and H. Hämmäinen, "Flexible spectrum management for mobile broadband services: How does it vary across advanced and emerging markets?" Telecommunications Policy, vol. 37, no. 2-3, pp. 178-191, 2013.

[4] M. Mustonen, M. Matinmikko, M. Palola, S. Yrjölä, and K. Horneman, "An evolution toward cognitive cellular systems: Licensed shared access for network optimization," IEEE Communications Magazine, vol. 53, no. 5, pp. 68-74, 2015.

[5] M. Gerasimenko, D. Moltchanov, R. Florea et al., "Cooperative radio resource management in heterogeneous cloud radio access networks," IEEE Access, vol. 3, pp. 397-406, 2015.

[6] F. Gomez-Cuba, F. J. Gonzalez-Castano, and J. Munoz-Castaner, "Is cooperative spectrum leasing by third-party relays advantageous in next-generation cellular networks?" in Proceedings of the 20th European Wireless Conference, pp. 1-7, VDE, 2014.

[7] Ericsson, Spectrum Sharing, Fast-Track Capacity with Licensed Shared Access, 2013.

[8] H. Gao, W. Ejaz, and M. Jo, "Cooperative Wireless Energy Harvesting and Spectrum Sharing in 5G Networks," IEEE Access, vol. 4, pp. 3647-3658, 2016.

[9] P. Spapis, K. Chatzikokolakis, N. Alonistioti, and A. Kaloxylos, "Using SDN as a key enabler for co-primary spectrum sharing," in Proceedings of the 5th International Conference on Information, Intelligence, Systems and Applications, IISA 2014, pp. 366371, Greece, July 2014.

[10] K. Buckwitz, J. Engelberg, and G. Rausch, "Licensed Shared Access (LSA) - Regulatory background and view of Administrations," in Proceedings of the 9th International Conference on Cognitive Radio Oriented Wireless Networks, CROWNCOM 2014, pp. 413-416, Finland, June 2014.

[11] N. Ul Hasan, W. Ejaz, N. Ejaz, H. S. Kim, A. Anpalagan, and M. Jo, "Network Selection and Channel Allocation for Spectrum Sharing in 5G Heterogeneous Networks," IEEE Access, vol. 4, pp. 980-992, 2016.

[12] P. Masek, E. Mokrov, A. Pyattaev et al., "Experimental evaluation of dynamic licensed shared access operation in live 3GPP LTE system," in Proceedings of the 59th IEEE Global Communications Conference, GLOBECOM 2016, USA, December 2016.

[13] I. Gudkova, E. Markova, P. Masek et al., "Modeling the utilization of a multi-tenant band in 3GPP LTE system with Licensed Shared Access," in Proceedings of the 8th International Congress on Ultra Modern Telecommunications and Control Systems and Workshops, ICUMT 2016, pp. 119-123, Portugal, October 2016.

[14] D. Guiducci, C. Carciofi, V. Petrini et al., "Sharing under licensed shared access in a LTE real test network at 2.3-2.4 GHz," in Proceedings of the 27th IEEE Annual International Symposium on Personal, Indoor, and Mobile Radio Communications, PIMRC 2016, Spain, September 2016.

[15] J. M. Peha, "Approaches to spectrum sharing," IEEE Communications Magazine, vol. 43, no. 2, pp. 10-12, 2005.

[16] M. Matinmikko, M. Mustonen, D. Roberson et al., "Overview and comparison of recent spectrum sharing approaches in regulation and research: From opportunistic unlicensed access towards licensed shared access," in Proceedings of the International Symposium on Dynamic Spectrum Access Networks (DYSPAN), pp. 92-102, IEEE, 2014.

[17] M. Palola, M. Matinmikko, J. Prokkola et al., "Live field trial of Licensed Shared Access (LSA) concept using LTE network 
in $2.3 \mathrm{GHz}$ band," in Proceedings of the IEEE International Symposium on Dynamic Spectrum Access Networks (DYSPAN '14), pp. 38-47, McLean, Va, USA, April 2014.

[18] V. Frascolla, M. M. Butt, N. Marchetti et al., "Dynamic Licensed Shared Access - A new architecture and spectrum allocation techniques," in Proceedings of the 84th IEEE Vehicular Technology Conference, VTC Fall 2016, Canada, September 2016.

[19] ECC, "Licensed Shared Access (LSA)," Report 205 Technical Report, 2014, http://www.erodocdb.dk/Docs/doc98/official/pdf/ ECCREP205.PDFt.

[20] A. Ponomarenko-Timofeev, A. Pyattaev, S. Andreev, Y. Koucheryavy, M. Mueck, and I. Karls, "Highly dynamic spectrum management within licensed shared access regulatory framework," IEEE Communications Magazine, vol. 54, no. 3, pp. 100109, 2016.

[21] E. Markova, I. Gudkova, A. Ometov et al., "Flexible Spectrum Management in a Smart City within Licensed Shared Access Framework," IEEE Access, 2017.

[22] B. Bahrak and J.-M. J. Park, "Coexistence decision making for spectrum sharing among heterogeneous wireless systems," IEEE Transactions on Wireless Communications, vol. 13, no. 3, pp. 1298-1307, 2014.

[23] C. Hernandez, C. Salgado, H. López, and E. RodriguezColina, "Multivariable algorithm for dynamic channel selection in cognitive radio networks," EURASIP Journal on Wireless Communications and Networking, vol. 2015, no. 1, article no. 216, 2015.

[24] I. Alqerm and B. Shihada, "Adaptive decision-making scheme for cognitive radio networks," in Proceedings of the 28th International Conference on Advanced Information Networking and Applications, pp. 321-328, IEEE, 2014.

[25] U. Mir and L. Nuaymi, "LTE pricing strategies," in Proceedings of the 77th Vehicular Technology Conference (VTC Spring), pp. 1-6, IEEE, 2013.

[26] E. Zeydan, A. S. Tan, I. A. Karatepe, A. S. Er, and G. Özcan, "Connectivity management using multiple attribute decision making in heterogeneous networks," in Proceedings of the 12th International Symposium on Wireless Communication Systems, ISWCS 2015, pp. 461-465, Belgium, August 2015.

[27] T. Martin and K.-C. Chang, "Assessing user decision behaviors for Dynamic Spectrum Sharing and pricing models," in Proceedings of 19th International Conference on Information Fusion (FUSION), pp. 1011-1018, ISIF, 2016.

[28] Y. Yang, L. T. Park, N. B. Mandayam, I. Seskar, A. L. Glass, and N. Sinha, "Prospect Pricing in Cognitive Radio Networks," IEEE Transactions on Cognitive Communications and Networking, vol. 1, no. 1, pp. 56-70, 2015.

[29] T. Çavdar, E. Güler, and Z. Sadreddini, "Instant overbooking framework for cognitive radio networks," Computer Networks, vol. 76, pp. 227-241, 2015.

[30] E. Mokrov, A. Ponomarenko-Timofeev, I. Gudkova, S. Andreev, and K. Samouylov, "Modeling a load balancing scheme between primary licensed and LSA frequency bands in 3GPP LTE networks," APTP + MS'2015, p. 54, 2015.

[31] I. F. Akyildiz, W. Lee, M. C. Vuran, and S. Mohanty, "NeXt generation/dynamic spectrum access/cognitive radio wireless networks: a survey," Computer Networks, vol. 50, no. 13, pp. 2127-2159, 2006.

[32] K. Ginevičius, "Normalization of quantities of various dimensions," Journal of Business Economics and Management, vol. 9, no. 1, pp. 79-86, 2008.
[33] T. L. Saaty and L. G. Vargas, Models, Methods, Concepts \& Applications of The Analytic Hierarchy Process, vol. 175, Springer Science Business Media, 2012.

[34] N. Bhushan and K. Rai, Strategic Decision Making: Applying The Analytic Hierarchy Process, Springer Science \& Business Media, 2007.

[35] M. Brunelli, Introduction to the Analytic Hierarchy Process, Springer, 2014. 


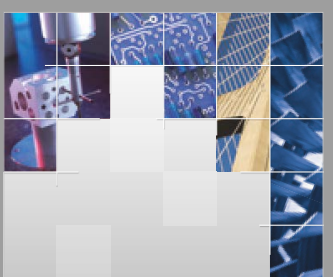

\section{Enfincering}
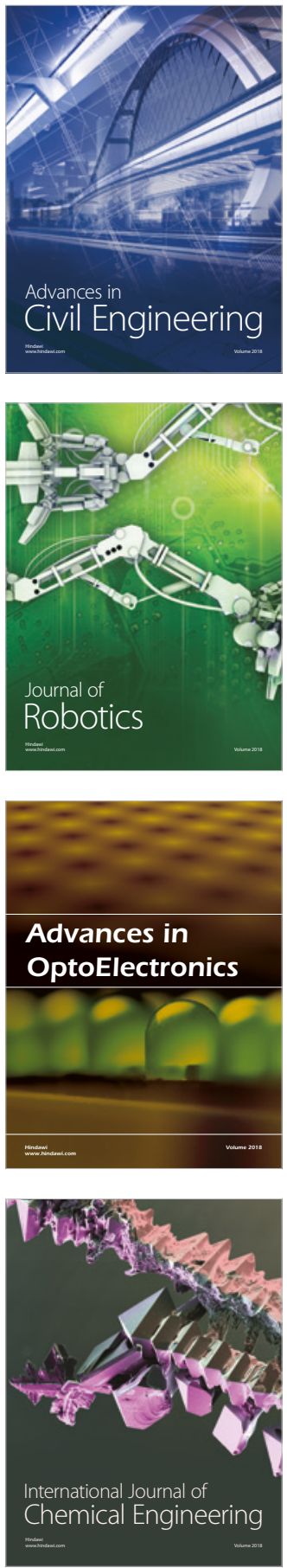

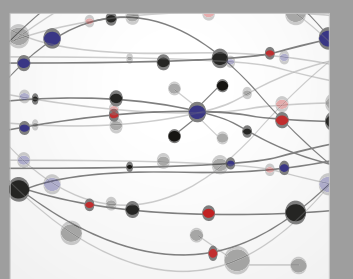

\section{Rotating \\ Machinery}

The Scientific World Journal

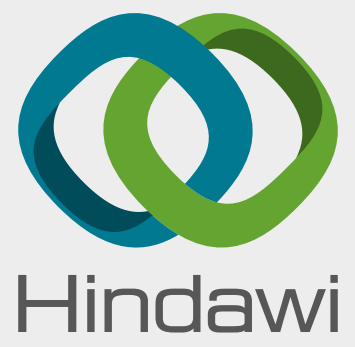

Submit your manuscripts at

www.hindawi.com
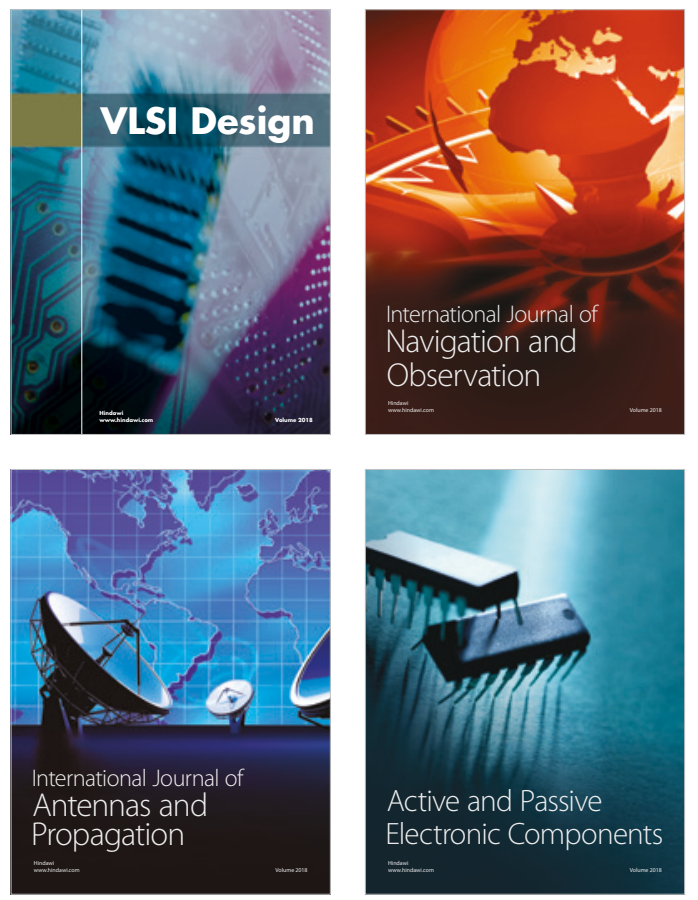
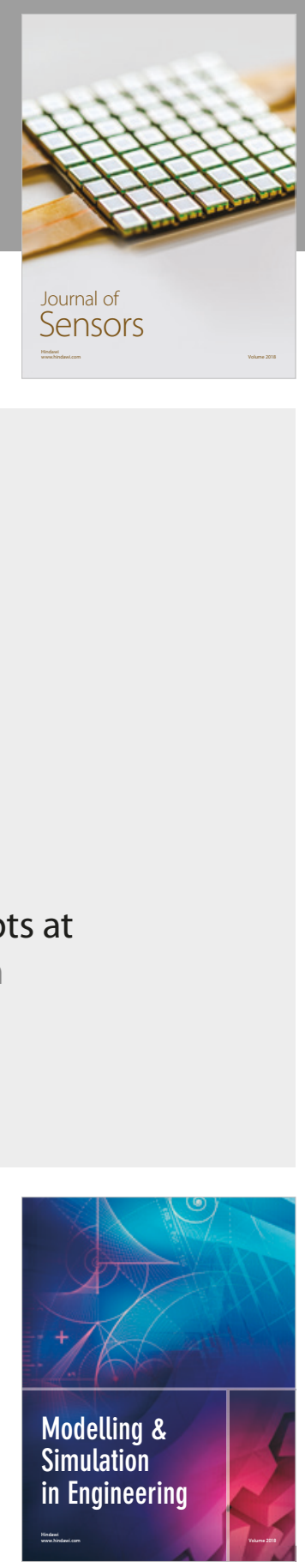

\section{Advances \\ Multimedia}
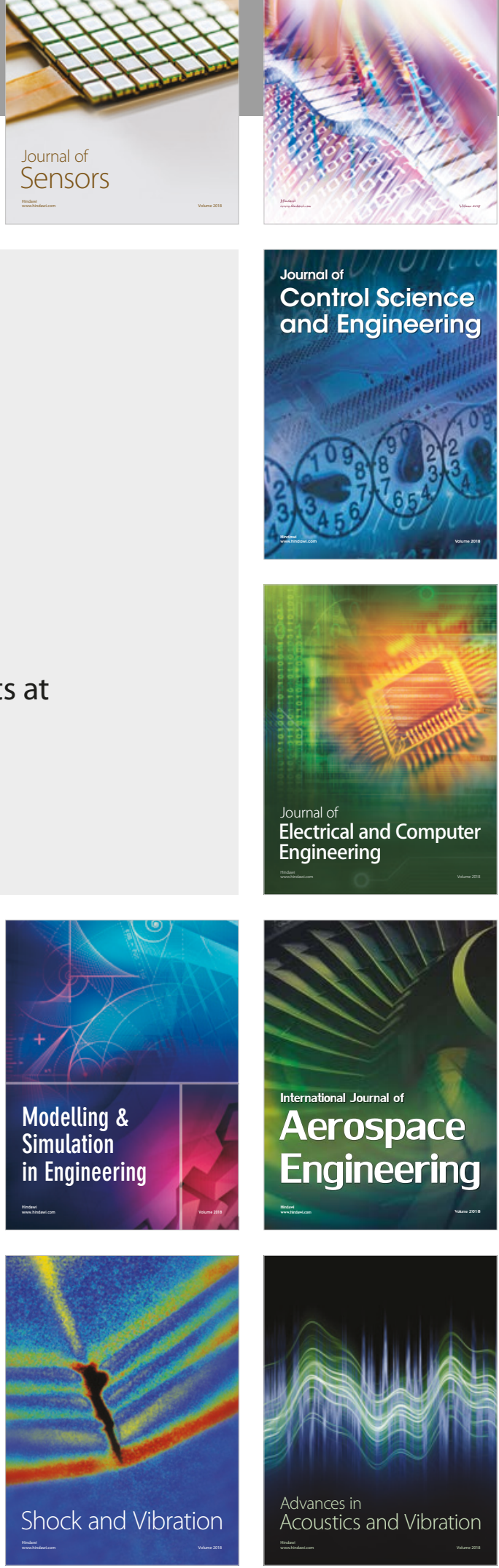